\title{
I5. COMMISSION DE LA ROTATION SOLAIRE
}

\section{Président: M. Newall, Director of the Solar Physics Observatory, Cambridge University, England.}

Membres: MM. Carrasco, Deslandres, De Lury, Evershed, Fox, Sampson, St John.

The interest in the subject of the rotation of the sun has undergone a change which has been brought about by the establishment of the 22-year sun-spot period derived from the observed peculiarities in the magnetic conditions of sun-spots in alternate half-cycles (II years). Attention is now concentrated upon a search for variation in the regime of the latitude-variation of angular velocity.

The observations made at Mount Wilson from I908 to I924 show a small but definite decrease in the equatorial velocity ( $\mathrm{St}$ John).

The question arises whether this decrease is the cause or the effect of the magnetic changes. It would seem almost worth while to repeat if possible from time to time the difficult research into the general magnetic field of the sun. The disclosure of a cyclical change in the intensity of the field might be expected to help towards an explanation of the change in the relative polarity of bipolar spots in successive half-cycles.

Reports received from the various observatories engaged in the observations of the solar rotation show that in general the collection of observational material is ahead of the laborious measurements of the photographs. Several observers hope to have results (either provisional or definitive) for presentation at the meeting of the Union at Leiden.

The question of variation of rotation with height above the photospheric layer appears to be now well established. The following tables summarise results in terms of linear velocities deduced from spectral lines attributed to medium levels (St John).

Change of Rotation with Level

A. Data from different sources.

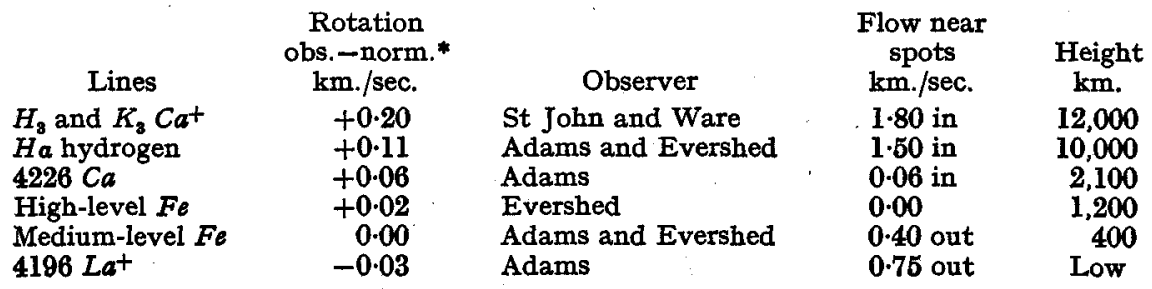

* Norm. =linear velocity for lines of medium level.

B. Simultaneous observations at high and low levels,

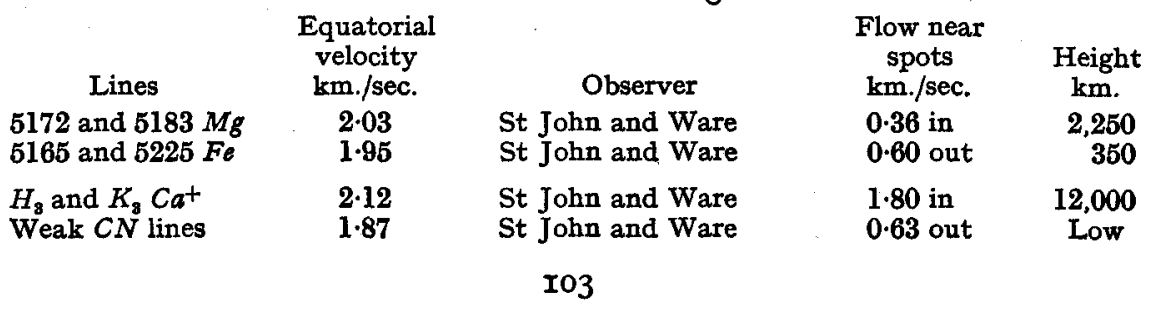


Recent results obtained by Evershed (M.N., R.A.S. 88, 126-r34) show marked differences between the angular velocities of prominences and those of sun-spots and the reversing layer, thus confirming his previous researches in the years Ig08-I9IX.

The daily angular motions $\xi$ derived from 45 east-limb spectra and 47 westlimb spectra, obtained during the years $1926-27$ are as follows:

$\begin{array}{cccc}\text { No. of spectra } & \text { Latitude } & \xi \text { prominences } & \xi \text { spots } \\ 33 & 8^{\circ} & 22^{\circ} \cdot 1 \pm 1^{\circ} \cdot 1 & 14^{\circ} \cdot 4 \\ 25 & 18 & 16 \cdot 6 \pm 0 \cdot 8 & 14 \cdot 1 \\ 26 & 25 & 19 \cdot 7 \pm 1 \cdot 4 & 13 \cdot 9 \\ 8 & 35 & 16 \cdot 8 \pm 1 \cdot 8 & 13 \cdot 5\end{array}$

The indication here shown of a polar retardation is perhaps real, although a considerable modification in the figures might doubtless result from a larger number of measures. The irregularities in $\xi$ are in large measure due to the eastern prominences, which were far more erratic in their movements than the western. As Mr Evershed has stated in a previous communication (M.N., R.A.S. 85, 607), the reversing layer, chromosphere and prominence regions are all disturbed by these irregular motions which appear to increase in amplitude with height above the photosphere. The most consistent values of the rotation are therefore obtained from the lowest levels in the reversing layer.

Amongst the suggestions received from members of the Commission are the following:

(a) In future researches regarding such questions as the variation of rotation with latitude or with the sun-spot period it would be desirable to choose for measurement lines representing the lowest levels in the reversing layer, either weak lines in the violet regions or stronger low-level lines in the red. (Evershed.)

(b) The differences between the values of the solar rotation rate measured previously to rgro and since that time should be investigated, inasmuch as some of the difference may be due to the method of measurement of the spectrograms.' Some of the old plates and some of the new might be passed (in negative copies) to different observers for comparative measurement. (De Lury.)

Preparations are being made at the Solar Physics Observatory, Cambridge, for the application of the methods of the interferometer to the study of spectroheliographic investigations. The results should be valuable for the study both of the general movements of the upper solar atmosphere and of local variations in the reversing layer.

The recommendations suggested in the reports communicated at Rome in I922 and at Cambridge in I925 are here repeated, as being still pertinent.

\section{Recommendations for Programme of Work}

(Repeated from the Rome Report, I922, and the Cambridge Report, I925)

(I) In order to trace to their source the systematic differences that are found in the value of the solar rotation by different observers, it is desirable that further study be given to possible sources of systematic errors and to the determination of the sources of the differences found by the same observer upon plates taken at frequent intervals, differences of the same order of magnitude as those between different observers.

(2) In the meantime, it is desirable to carry on, when possible, continuous 
series of observations under constant instrumental conditions, and by the same observer.

(3) As it seems increasingly probable that local conditions in the reversing layer are frequent causes of divergent results, it is advisable to extend observations over a considerable period of time in investigations of such questions as variation with latitude or differences between hemispheres.

(4) Simultaneous observation on the centre and the limb is recommended as a valuable means of checking results, and of investigating the hemispheres separately.

I3 March I928

H. F. NEWALL

President of the Commission 